\title{
Taxonomic integrative and phylogenetic identification of the first recorded Triatoma rubrofasciata in Zhangzhou, Fujian Province and Maoming, Guangdong Province, China
}

\author{
Yue Hu ${ }^{1,2,3+}$, Min-Zhao Gao ${ }^{4 \dagger}$, Ping Huang ${ }^{1,2,3}$, Hong-Li Zhou ${ }^{1,2,3}$, Yu-Bin Ma 1,2,3, Min-Yu Zhou ${ }^{1,2,3}$,
} Shao-Yun Cheng ${ }^{1,2,3}$, Han-Guo Xie $5^{5^{*}}$ and Zhi-Yue LV $\mathrm{LV}^{1,2,3^{*}}$

\begin{abstract}
Background: Most species of Triatominae live exclusively in Latin America. However, one species, Triatoma rubrofasciata, has been recorded in the Americas as well as in various port areas in Africa and Asia. An increasing number of T. rubrofasciata have been reported in southern China in recent years. However, the origin of this invasive insect vector in China remains unknown, therefore, accurate identification and phylogenetic analysis of the bugs are urgently needed.

Methods: A total of seven triatomine insect specimens were found and collected from Maoming City, Guangdong Province, China (GDMM) and Zhangzhou City, Fujian Province, China (FJZZ), respectively. The obtained insect vector specimens were observed under a dissecting microscope for morphological classification and then the genomic DNA was extracted, and the $16 \mathrm{~S}$ ribosomal RNA (rRNA), $28 \mathrm{~S}$ rRNA as well as cytochrome oxidase subunit I (COI) genes of the species were amplified and sequenced. Subsequently, molecular phylogenetic analyses based on multiple alignments of the above genes were conducted in order to identify the species and determine the phylogenetic origin approximation accurately.

Results: The triatomine insects collected from GDMM and FJZZ were identified as Triatoma rubrofasciata using morphological and genetic analyses. All of the Chinese T. rubrofasciata captured in FJZZ, GDMM and other localities in southern China, together with a Vietnamese and Brazilian strain, formed a new, cohesive clade. T. rubrofasciata in GDMM and FJZZ are likely derived from strains found in Vietnam or Brazil.

Conclusions: To the best of our knowledge, this is the first record of the invasive insect T. rubrofasciata, which is likely derived from strains native to Vietnam or Brazil, in both Maoming City, Guangdong Province and Zhangzhou City, Fujian Province of China. A comparison of the DNA sequences of the $16 \mathrm{~s}$ rRNA, $28 \mathrm{~s}$ rRNA and COI genes confirmed the specific identification of T. rubrofasciata, and its potential origin in China is based on the phylogenetic analyses undertaken in this study. More targeted interventions and improved entomological surveillance are urgently needed to control the spread of this haematophagous insect in China.
\end{abstract}

Keywords: Triatoma rubrofasciata, Molecular identification, Phylogenetic study

\footnotetext{
*Correspondence: xiehanguo@163.com; Ivzhiyue@mail.sysu.edu.cn

†Yue Hu and Min-Zhao Gao are the joint first authors.

${ }^{5}$ Fujian Center for Disease Control and Prevention, Fuzhou 350001, China

'Zhongshan School of Medicine, Sun Yat-sen University, Guangzhou 510080,

China

Full list of author information is available at the end of the article
}

(c) The Author(s). 2019 Open Access This article is distributed under the terms of the Creative Commons Attribution 4.0 International License (http://creativecommons.org/licenses/by/4.0/), which permits unrestricted use, distribution, and reproduction in any medium, provided you give appropriate credit to the original author(s) and the source, provide a link to the Creative Commons license, and indicate if changes were made. The Creative Commons Public Domain Dedication waiver (http://creativecommons.org/publicdomain/zero/1.0/) applies to the data made available in this article, unless otherwise stated. 


\section{Multilingual abstracts}

Please see Additional file 1 for translations of the abstract into the five official working languages of the United Nations.

\section{Background}

Triatomines, also known as kissing bugs due to their tendency to bite human faces, are insects from the order Hemiptera and family Reduviidae; they are the main vectors responsible for the transmission of American trypanosomiasis (Chagas disease), a chronic, systemic, tropical parasitic disease caused by Trypanosoma cruzi that infects an estimated 8 million people worldwide, mostly in Latin America [1-3]. There are a total of 151 triatomine species (149 extant and two extinct) that are currently composed of five tribes: Aberproseniini, Bolboderini, Cavernicolini, Rhodniini and Triatomini. Most Triatomine occur in the New World; only one genus, Triatoma, which is included in the Triatomini, is found in both in the Old and New Worlds [3, 4].

Triatoma rubrofasciata is characterized by a triangular scutellum and an orange-red margin along the outer edge of the abdomen and the sides of the pronotum [5]; it is a cosmopolitan species from the genus Triatoma and is widely distributed across the globe, although it is most commonly found in Asia, Oceania, Africa and Central America [6-8]. This tropicopolitan insect is also widely spread across the southern coastal areas of China including Guangdong, Guangxi, Hainan and Taiwan [6, 9]. Although Chagas disease has not been reported in China, dermatitis and anaphylactic shock caused by triatomine insects have been recorded since the 1980s [10, 11]. Trends in global vector migrations from endemic to non-endemic countries have greatly increased the risk of Chagas disease expansion to global levels [12]; migration of these insect vectors could help spread this disease beyond its previous limits [13], which suggests there is a potential risk of Chagas disease being introduced and transmitted in China, posing a significant threat to public health [14]. Therefore, accurate identification and phylogenetic analysis of T. rubrofasciata will trace back its potential origin and carry out scientific and effective prevention, which may helpful for the control of its spread.

The distinctive features of the triatomine are based primarily on morphological characteristics such as the genitalia $[15,16]$ and appendages $[17,18]$, although molecular markers have also been used. However, morphological identification is complicated, time-consuming and sometimes inaccurate, especially for specimens in nymphal stages, due to the wide variety of triatomines (over 140 species). Recently, with the rapid development of molecular biology techniques and bioinformatics, molecular identification based on specific DNA sequence analyses has become a common technological tool used to identify new species $[19,20]$, to provide rapid diagnoses of invasive pathogens [21] and cases of rare diseases [22], and to help in understanding the phylogenetic and phylogeographic relationships among species [23]. DNA markers including mitochondrial $16 \mathrm{~S}$ and nuclear $28 \mathrm{~S}$ ribosomal RNA (rRNA), mitochondrial cytochrome oxidase subunit I (COI), as well as DNA markers of nuclear origin, such as the internal transcribed spacer 2 (ITS-2) have been used to analyse genetic variation among triatomines, and explain their population structure and evolutionary history [6, 10, 23-28]. Therefore, this paper aims to use the above three universal markers for the identification of triatomine species found in Maoming, Guangdong Province and Zhangzhou, Fujian Province, China and to trace their potential origin through phylogenetic analysis.

\section{Materials and methods}

Insect collection and morphological identification

Triatomine insects were actively searched for and caught using a butterfly net by technicians from Fujian Centre for Disease Control and Prevention, China. Captures took place in local residents' houses close to each other in Huping Village (E 117 $38^{\prime}, \mathrm{N} 24^{\circ} 39^{\prime}$ ), Fengshan Town, Hua'an County, Zhangzhou City, Fujian Province, China (FJZZ) in August 2017. Additionally, Dr. Min-Zhao Gao made captures in one dwelling (under wooden beds) in Tiantou Village (E $111^{\circ} 02^{\prime}, \mathrm{N}$ $\left.21^{\circ} 33^{\prime}\right)$, Shuidong Town, Dianbai District, Maoming City, Guangdong Province, China (GDMM) in August 2018 (Fig. 1). The locations chosen for collection were based on oral reports from local residents who found the insects in their houses. Triatomines were captured and transported live to a laboratory and identified using morphological features under a stereoscopic microscope (SZ650, Cnoptec, Chongqing, China) as described previously [29].

\section{DNA extraction, amplification, cloning and sequencing}

Total genomic DNA was isolated from three legs of each triatomine specimen using the HiPure Tissue DNA Micro Kit (Cat No. D3125-02, Magen, Guangzhou, China) according to the manufacturer's recommended protocol. The genes of mitochondrial 16S rRNA, COI and nuclear 28S rRNA were amplified using primers synthesized by Sangon Biotech (Shanghai, China), which are listed in Table 1.

Polymerase chain reactions (PCR) were performed in $50 \mu \mathrm{L}$ of the following mixture: approximately 30 ng of DNA template, $1.1 \times$ Golden Star T6 Super PCR Mix (Tsingke, China) and $0.4 \mu \mathrm{mol} / \mathrm{L}$ of each primer 


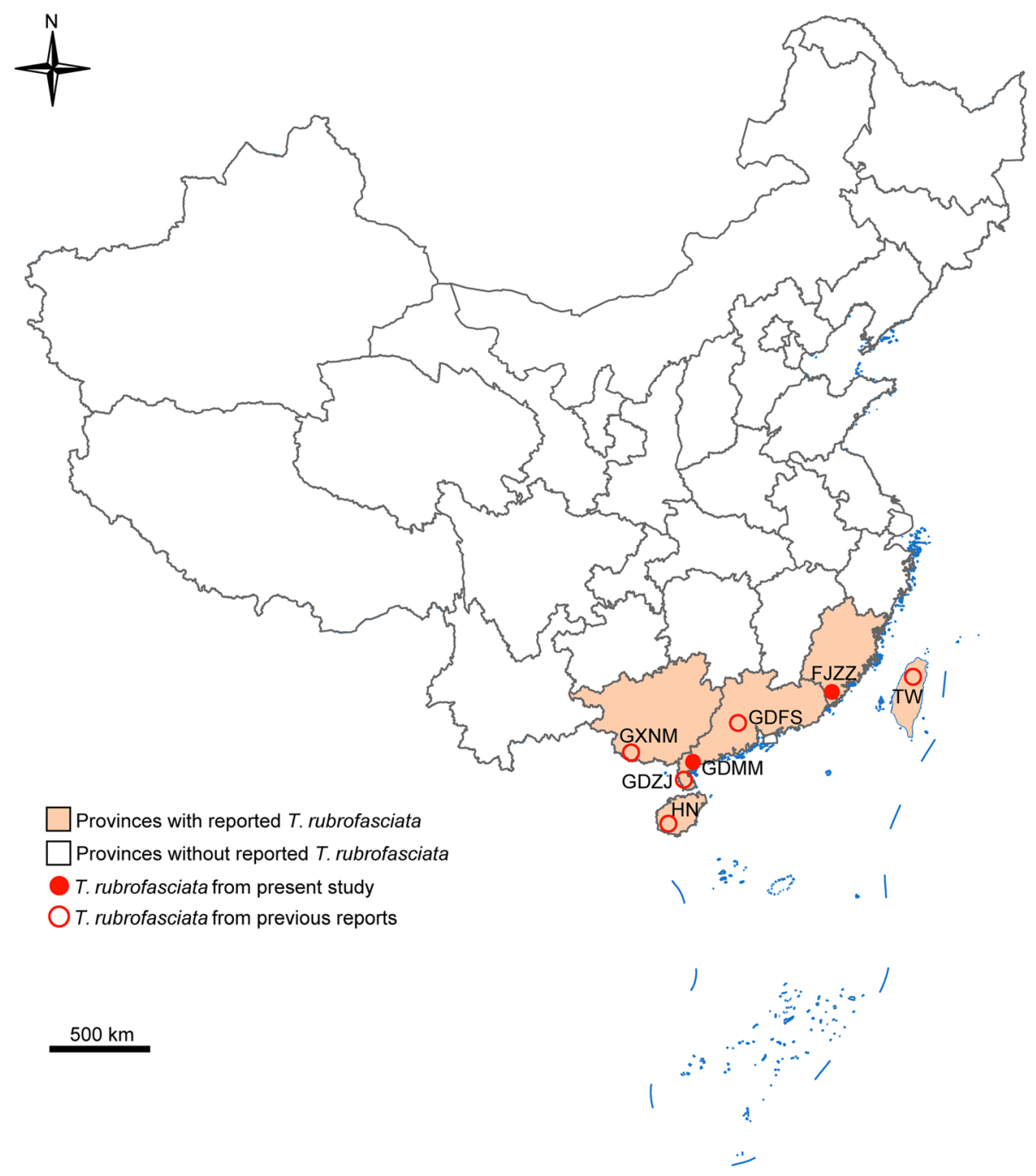

Fig. 1 Map of distribution of Triatoma rubrofasciata caught in China in the present study or the previous reports. FJZZ: Zhangzhou City, Fujian Province; GDFS: Foshan City, Guangdong Province; GDMM: Maoming City, Guangdong Province; GDZJ: Zhanjiang City, Guangdong Province; GXNM: Ningming County, Guangxi Province; HN: Hainan Province; TW: Taiwan

using a Bio-Rad PCR C1000 Touch instrument (BioRad, USA). Negative controls were made with ultrapure water to control for contamination during PCR. For the 16S rRNA, 28S rRNA and COI genes, the fragments were amplified using the following thermal cycling conditions: initial denaturation at $98^{\circ} \mathrm{C}$ for 2 min; 35 cycles of denaturation at $98^{\circ} \mathrm{C}$ for $10 \mathrm{~s}$, annealing at $55^{\circ} \mathrm{C}$ for $30 \mathrm{~s}$, extension at $72{ }^{\circ} \mathrm{C}$ for $15 \mathrm{~s}$ and final extension at $72{ }^{\circ} \mathrm{C}$ for $3 \mathrm{~min}$. Afterwards, the
PCR products were separated using $1 \%$ agarose gel electrophoresis, and the expected bands were excised under ultraviolet light, followed by purification with an Agarose Gel DNA Extraction Kit (Takara, Japan). The purified DNA fragments were inserted into a pClone007 Blunt Simple Vector (Tsingke, China), and the plasmids were used to transform E. coli DH5a. Sequencing was performed using the vector primers (M13 forward and M13 reverse) in an Automated

Table 1 Primers used to amplify the mitochondrial 16S rRNA, COI genes and the nuclear ribosomal 28S rRNA gene

\begin{tabular}{llll}
\hline Gene target & Forward primer $\left(5^{\prime} \rightarrow 3^{\prime}\right)$ & Reverse primer $\left(5^{\prime} \rightarrow 3^{\prime}\right)$ & Expected length $(\mathrm{bp})$ \\
\hline 16S rRNA & GGTTTGAATGGCCGCAGTA & TCTCCGGTTTGACTCAGATCA & 491 \\
28S rRNA & GCGAGTCGTGTTGCTTGATAGTGCAG & TTGGTCCGTGTTTCAAGACGGG & 678 \\
\hline
\end{tabular}


DNA Analyzer (ABI 3730XL, Applied Biosystems, Foster City, CA, USA) with the BigDye Terminator v3.1 Cycle Sequencing Kit (Cat No. 4337457, Applied Biosystems, Foster City, CA, USA) by Tsingke Biotechnology Ltd., Co. (Guangzhou, China).

\section{Sequence alignment and phylogenetic analysis}

The sequences of the 16S rRNA, 28S rRNA and COI genes were submitted to the National Centre for Biotechnology Information (NCBI) GenBank database (https://www.ncbi.nlm.nih.gov/genbank/) under the accession numbers MK601647, MK601646, MK602653, MK602652, MK614012 and MK614011, respectively. They were submitted to GenBank for homology searches subsequent to triatomine species classification using BLAST (http://blast.ncbi.nlm.nih.gov/Blast. cgi) [30]. For the three genes, multiple alignments were performed among the best hit sequences using ClustalW2 [31] with default parameters; phylogenetic trees were constructed with MEGA 7.0 [32] using the Maximum Likelihood (ML) method [10] based on the Kimura 2-Parameter (K2P) nucleotide substitution model [33] and assessed with the bootstrap-resampling technique over 1000 replications. To demonstrate the polymorphism of $T$. rubrofasciata from multiple localities and their intraspecific genetic divergence, the pairwise genetic distances of $16 \mathrm{~S}$ rRNA, $28 \mathrm{~S}$ rRNA and COI were calculated between sequences obtained in this study and reference sequences downloaded from GenBank using the K2P nucleotide substitution model in MEGA 7.0 (www. megasoftware.net) [32].

\section{Results}

\section{Morphological characteristics of Triatoma rubrofasciata}

Four adult triatomine insects (two males and two females) were caught on a balcony where lumber was stored on the second floor of two farmers' dwellings at Huping Village, Fengshan Town, Hua'an County (FJZZ). In addition, three adult triatomine insects (two males and one female) were captured under wooden beds in a living-room on the second floor of a local resident's house at Tiantou Village, Shuidong Town, Dianbai District (GDMM).

The triatomines collected in FJZZ and GDMM showed the morphological characteristics of $T$. rubrofasciata (Figs. 2 and 3). The specimens were black with orangered margins along the lateral edge of the pronotum, wings and abdomen. The forepart of the pronotum was narrow, whereas the posterior was wide. The female and the adult males were approximately $23 \mathrm{~mm}$ and $21 \mathrm{~mm}$ in length respectively, with slightly elliptical shapes, and the long, cone-shaped heads were approximately three millimeter in length (Fig. 2). The thread-like antennae, located between the black compound eyes and clypeus, consisted of four segments, with the 1st segment surpassing the apex of head (Fig. 3a, b). Numerous small particles were densely distributed around the head and pronotum (Fig. 3a). The scutellum was triangular in shape with wrinkles in the middle and a sharp, needlelike apex (Fig. 3a). The genital segment of the adult female was triangular form, while that of the adult male had a broad circular shape (Fig. 3c, d).

\section{Molecular identification and phylogenetic relationships of Triatoma rubrofasciata}

In this study, apart from morphological identification, we also used a molecular identification method based on sequences of $16 \mathrm{~S}$ and $28 \mathrm{~S}$ rRNA as well as COI to classify the triatomine species from GDMM and FJZZ. The results of the similarity analyses on the basis of the above genes demonstrated that the triatomine species in these two localities were accurately identified as $T$. rubrofasciata with $96-100 \%$ sequence matching identities.

For the 16S rRNA gene, T. rubrofasciata from FJZZ displayed the highest similarity with specimens from Foshan City, Guangdong Province, China (GDFS) with 99\% identity. T. rubrofasciata from GDMM demonstrated the highest similarity with $T$. rubrofasciata from Zhanjiang City, Guangdong Province, China (GDZJ) with $99 \%$ identity. A polymorphism analysis of the $16 \mathrm{~S}$ rRNA gene showed that two transition sites differed between the sequences of the insects from FJZZ and GDMM, and from FJZZ and GDZJ. Similarly, two distinctive transition sites were observed between the $16 \mathrm{~S}$ rRNA sequences of the insects from GDMM and GDFS. Furthermore, one transversion site and one transition site differed between the 16S rRNA sequences of $T$. rubrofasciata from FJZZ and TW, while one transversion site and three transition sites differed between the sequences of the insects from GDMM and TW. However, only one distinguishing transition site was detected between the sequences of the insects from FJZZ and Vietnam and from GDMM and Vietnam. The genetic distance of the 16S rRNA gene ranged from 0.000 to 0.003 in T. rubrofasciata from the different locations, and the average intraspecific distance was 0.001 (Table 2, Additional file 2: Table S1).

For the $28 \mathrm{~S}$ rRNA gene, both T. rubrofasciata from FJZZ and GDMM were similar, with T. rubrofasciata from GDZJ demonstrating 100\% identity. The 28S rRNA sequences of $T$. rubrofasciata from FJZZ and GDMM were identical and only one distinctive transversion site was found between the sequences of $T$. rubrofasciata from FJZZ and GDFS, whereas no sites differed between the sequences of $T$. rubrofasciata from FJZZ and Ningming County, Guangxi Province, China (GXNM). 

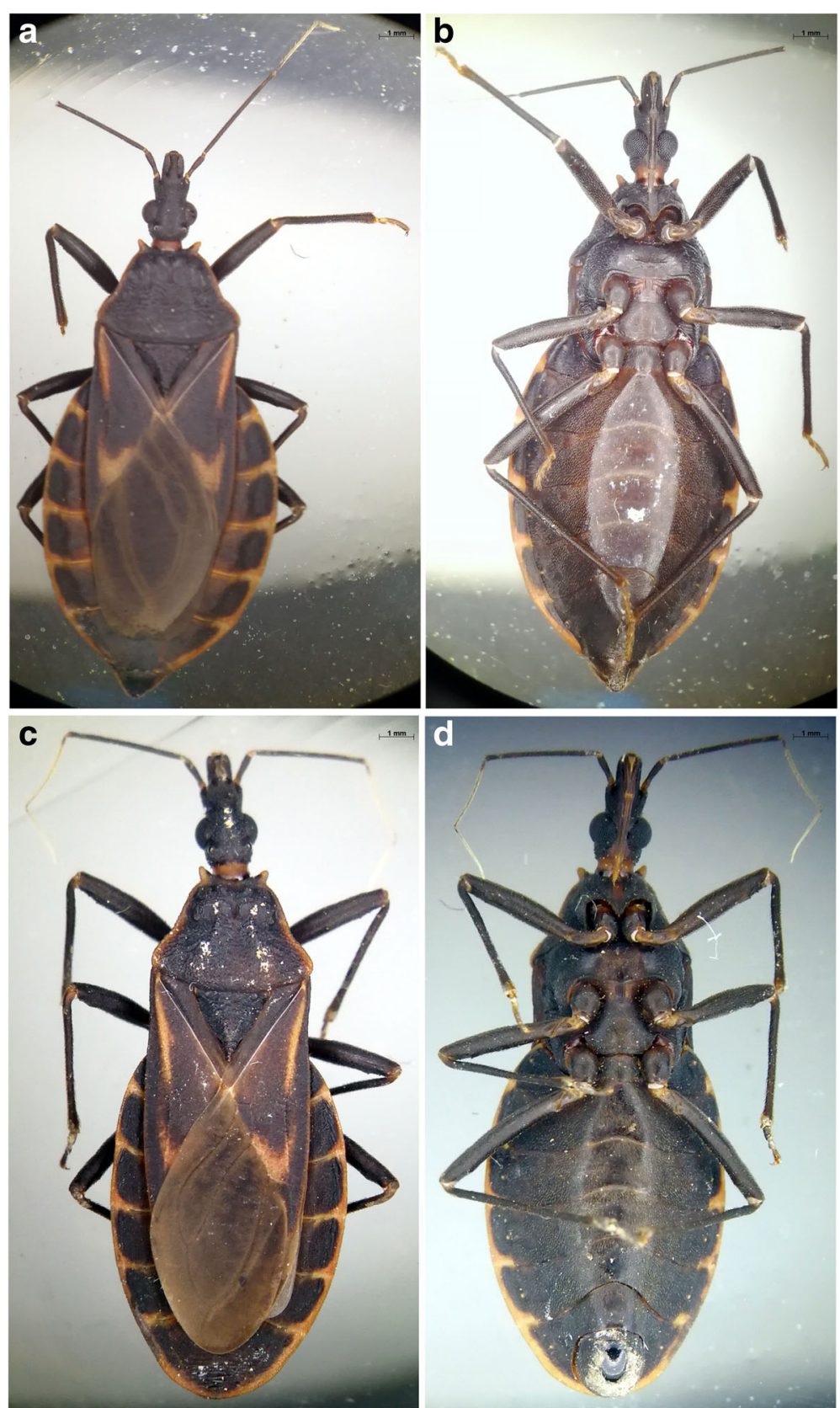

Fig. 2 Dorsal and ventral views of the female ( $\mathbf{a}$ and $\mathbf{b}$ ) and male (c and $\mathbf{d}$ ) adults of Triatoma rubrofasciata

In addition, two different transversion sites and one distinguishing transition site were found between the sequences of T. rubrofasciata from FJZZ and Brazil, while two transversion sites differed between the sequences of the triatomine insects from FJZZ and Vietnam. The genetic distance of the 28S rRNA gene in $T$. rubrofasciata from the different locations ranged from 0.000 to 0.002 , and the average intraspecific distance was 0.001 (Table 3, Additional file 3: Table S2).

The COI gene of T. rubrofasciata from both FJZZ and GDMM presented the highest similarity with that from
Brazil with $96 \%$ identity, with one variable site between them. Nevertheless, eighteen variable sites were detected between the COI sequences of the insects from FJZZ and Brazil, while nineteen variable sites were observed between the sequences of the insects from GDMM and Brazil. Thus, the genetic distance of the COI gene varied from 0.003 to 0.048 , and the mean intraspecific distance was 0.032 (Table 4, Additional file 4: Table S3).

Furthermore, phylogenetic analysis of triatomine species based on the sequences obtained in this study and reference sequences with high similarity obtained from GenBank for 

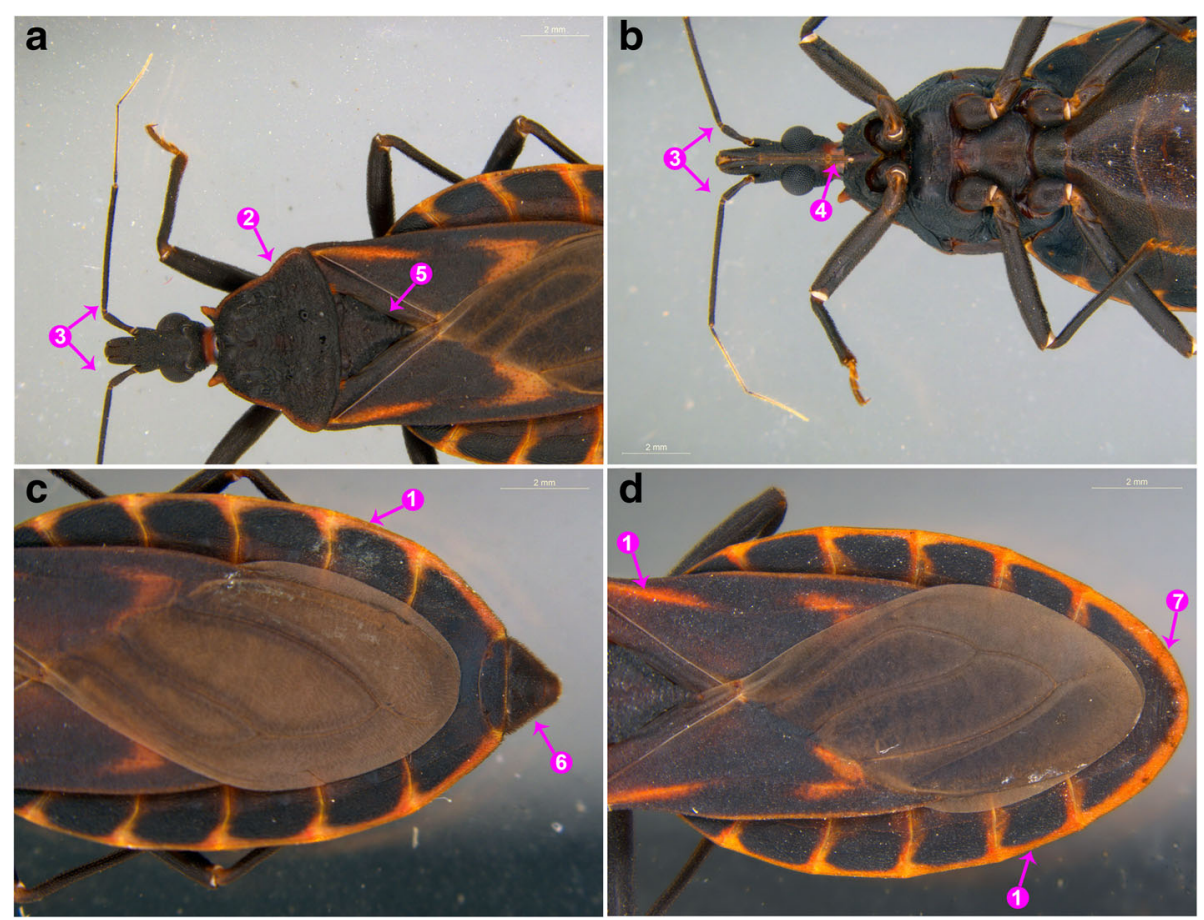

Fig. 3 The key morphological characteristics of Triatoma rubrofasciata. $\mathbf{a}$ and $\mathbf{b}$ : Dorsal and ventral views of the head and thorax of Triatoma rubrofasciata; c: Dorsal view of the abdomen of the female Triatoma rubrofasciata; $\mathbf{d}$ : Dorsal view of the abdomen of the male Triatoma rubrofasciata. (1): Orange-red margin along the outer edge of abdomen and wings; (2): Orange-red margin along the side of pronotum; (3): The antennae were located between the compound eyes and clypeus while the 1st segment of antennae surpassed the apex of head; (4): Mouthpart with a long and straight proboscis covered with short hairs and the hairs became longer towards the tip; (5): The scutellum was broad and triangular to the tip; (6): The genital segment of female adult appeared in triangular shape; (7): The genital segment of male adult was in a broad circular shape

the 16S rRNA, 28S rRNA and COI genes showed a distinct clade for all T. rubrofasciata individuals and was strongly supported by bootstrap values as high as 97 to $100 \%$, which also confirmed that the insects captured in FJZZ and GDMM were T. rubrofasciata (Figs. 4, 5 and 6). Moreover, these results indicate that the triatomine species in the ML phylogenetic trees might be identified accurately using the barcode region used in this study.

According to the phylogenetic trees inferred from the $16 \mathrm{~S}$ rRNA and $28 \mathrm{~S}$ rRNA genes (Figs. 4 and 5), the Triatomini tribe is scattered throughout 4 main clades: (1) Clade A: South American Triatoma, including the brasiliensis subcomplex (T. brasiliensis from Brazil), the rubrovaria subcomplex (T. rubrovaria and $T$. circummaculata from Argentina), the infestans subcomplex ( $T$. infestans from Argentina) and the matogrossensis subcomplex (T. matogrossensis and T. costalimai from Brazil); (2) Clade B: genus Panstrongylus $(P$. geniculatus and $P$. megistus from Argentina, and P. lignarius from Brazil); (3) Clade C: North and Central American Triatoma, including the protracta complex, ( $T$. protracta from the United

Table 2 Pairwise intraspecific genetic distances of 165 rRNA gene of Triatoma rubrofasciata, based on the Kimura 2-Parameter model

\begin{tabular}{llllll}
\hline & GDZJ & GDFS & GDMM & FJZZ & TW \\
\hline GDZJ, China (MG674717) & - & & & & \\
GDFS, China (KY420176) & 0.000 & - & & & \\
GDMM, China (MK601646) & 0.000 & 0.000 & - & & - \\
FJZZ, China (MK601647) & 0.000 & 0.000 & 0.000 & - & 0.000 \\
TW, China (KP899112) & 0.000 & 0.000 & 0.000 & 0.003 & 0.003 \\
Vietnam (HQ337019) & 0.003 & 0.003 & 0.003 & - \\
\hline
\end{tabular}


Table 3 Pairwise intraspecific genetic distances of 285 rRNA gene of Triatoma rubrofasciata, based on the Kimura 2-Parameter model

\begin{tabular}{|c|c|c|c|c|c|c|c|}
\hline & GDZJ & GDFS & GDMM & FJZZ & GXNM & Vietnam & Brazil \\
\hline GDZJ, China (MG675575) & - & & & & & & \\
\hline GDFS, China (KY420177) & 0.000 & - & & & & & \\
\hline GDMM, China (MK602652) & 0.000 & 0.000 & - & & & & \\
\hline FJZZ, China (MK602653) & 0.000 & 0.000 & 0.000 & - & & & \\
\hline GXNM, China (MH356281) & 0.000 & 0.000 & 0.000 & 0.000 & - & & \\
\hline Vietnam (KR632547) & 0.000 & 0.000 & 0.000 & 0.000 & 0.000 & - & \\
\hline Brazil (KR632546) & 0.002 & 0.002 & 0.002 & 0.002 & 0.002 & 0.002 & - \\
\hline
\end{tabular}

GDZJ Zhanjiang City, Guangdong Province, GDFS Foshan City, Guangdong Province, GDMM Maoming City, Guangdong Province, FJZZ Zhangzhou City, Fujian Province, GXNM Ningming County, Guangxi Province

States of America), the phyllosoma subcomplex ( $T$. picturata from Mexico) and the dimidiata subcomplex ( $T$. dimidiata from Belize); and (4) Clade D: South American and Asian Triatoma, consisting of the rubrofasciata complex ( $T$. rubrofasciata from China, Vietnam and Brazil). All of the Chinese triatomines collected from GDFS, GDZJ, GDMM, FJZZ and TW, along with the Vietnamese strain, were classified in the rubrofasciata complex and merged into Clade D, forming a distinctive clade with the rubrofasciata complex from Brazil, as shown in Fig. 5. This strongly suggests a close relationship between Asian triatomines and the rubrofasciata complex from Brazil. Similarly, phylogenetic analysis of Triatominae COI sequences demonstrated that Chinese triatomines from FJZZ and GDMM and the Brazilian T. rubrofasciata were in the same clade (Fig. 6). The Triatomini tribe was scattered throughout three clades of the South American Triatoma (genus Panstrongylus) as well as the South American and Asian Triatoma, due to the limited sequences of the COI gene available in GenBank (Fig. 6). These results strongly imply that Vietnam or Brazil is the most likely origin of the $T$. rubrofasciata in China.

\section{Discussion}

T. rubrofasciata is a widespread haematophagous insect $[34,35]$, and it is the vector for $T$. cruzi, which causes Chagas disease and is a potential vector of bacteria and viruses [36]. It is likely distributed via shipping lines from the tropics and sub-tropics [34, 37, 38], and from

Table 4 Pairwise intraspecific genetic distances of $\mathrm{COI}$ gene of Triatoma rubrofasciata, based on the Kimura 2-Parameter model

\begin{tabular}{llll}
\hline & GDMM & FJZZ & Brazil \\
\hline GDMM, China (MK614011) & - & & \\
FJZZ, China (MK614012) & 0.003 & - & \\
Brazil (GQ869655) & 0.048 & 0.045 & - \\
\hline
\end{tabular}

GDMM Maoming City, Guangdong Province, FJZZ Zhangzhou City, Fujian Province inland regions in north-east India, Vietnam [35] and north-east Brazil [39]. Though T. rubrofasciata is the first species of the triatomine to have been described, its origins remain a mystery [40]. Several researchers have assumed an Old World (oriental) origin for T. rubrofasciata $[29,41]$. However, more recent studies have suggested that all Triatominae are of a New World origin and that they were transported in association with rats (Rattus norvegicus) on ships sailing from the Americas [42-44].

Clear identification of invasive species is crucial for ecosystem maintenance, control, and risk assessment [19]. In this paper, the triatomine were identified as $T$. rubrofasciata by morphological analysis, which is supported by both the genetic and phylogenetic species concepts, indicating that $16 \mathrm{~S}$ rRNA, $28 \mathrm{~S}$ rRNA and COI can be used as specific gene markers for the reliable identification of triatomine species, as suggested by previous studies $[6,10,44]$. Morphological taxonomy and molecular identification are the two primary methods used for the identification of species [45-47]. Morphological similarities at both the intra- and inter-species level limit the usefulness of morphological taxonomic keys in some species [48]. Moreover, molecular identification might be effectively applied to classify the immature stages of triatomines, as traditional taxonomic methods are difficult to use when distinguishing nymphs, especially considering the species complexes that exhibit large morphological and chromatic variation [28]. However, molecular identification will not take the place of morphological systematics because it relies on species definitions produced from morphological analyses [49]; hence, both approaches should be applied to provide effective, efficient and accurate species identifications $[50,51]$, and the integration of morphological and molecular methods is indicated for species recognition of T. rubrofasciata in future entomological surveys in China [6].

To determine genetic variation in T. rubrofasciata from the different sites, genetic distances were calculated. The polymorphism analyses results showed that transitions 


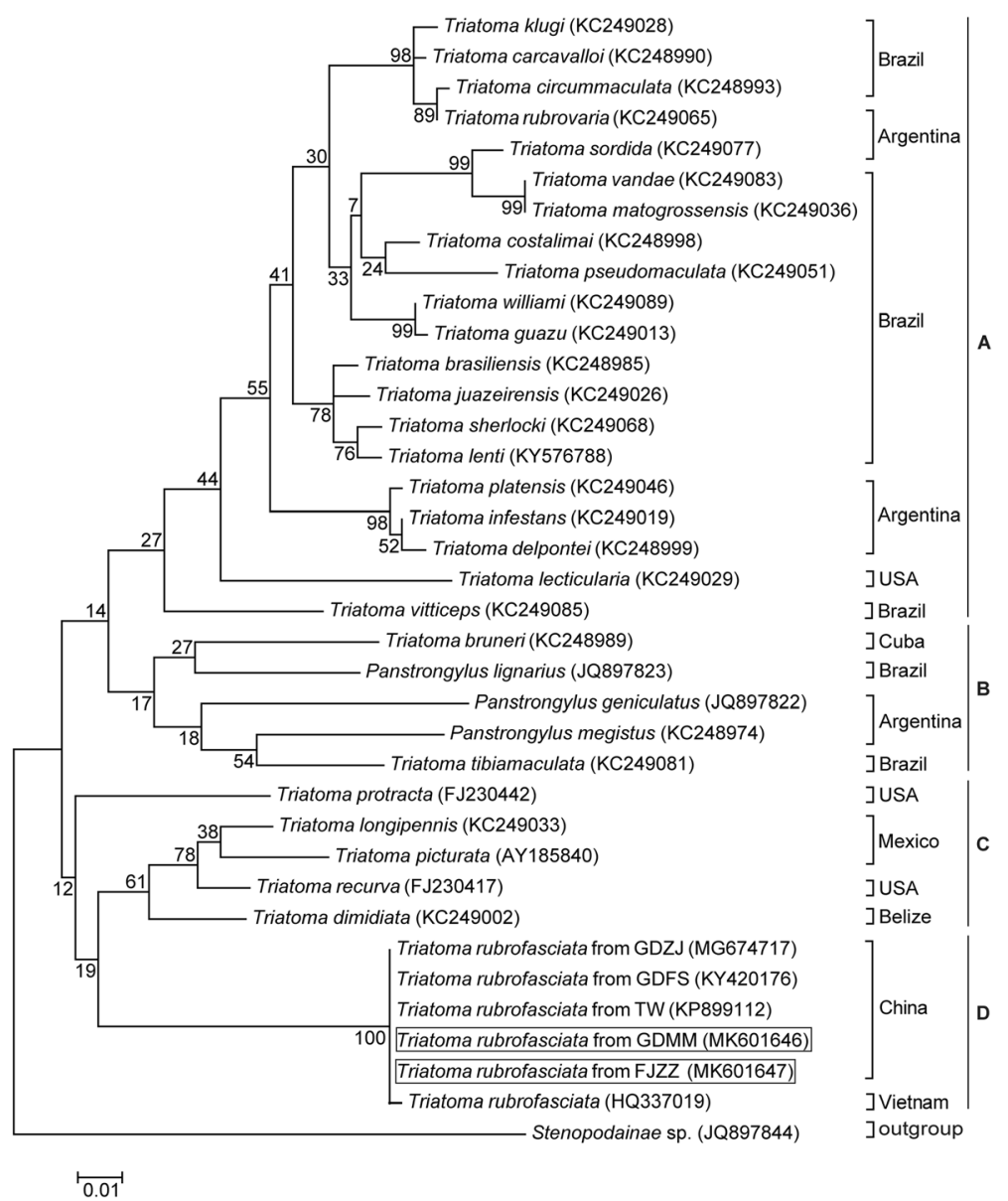

Fig. 4 Phylogenetic tree of 165 rRNA gene among triatomine species. The tree was constructed by using the Maximum Likelihood method based on the Kimura 2-Parameter model. The relative values (\%) on branches are based on 1000 bootstrap resamplings. Stenopodainae sp. was used to form the outgroup. T. rubrofasciata identified in the present study were shown in the square frame

were more frequent than transversions in the $16 \mathrm{~S}$ rRNA, $28 \mathrm{~S}$ rRNA and COI genes, and our data was consistent with observations from previous studies suggesting that $\mathrm{COI}$ is a faster evolving sequence, exhibiting higher variability than $16 \mathrm{~S}$ or $28 \mathrm{~S}$ rRNA [52, 53]. Hence, we suggest that $16 \mathrm{~S}$ rRNA, 28S rRNA as well as COI genes are suitable for species identification while for tracking back the origin of the species, COI gene is applicable due to the higher genetic diversity.

Despite a relevant body of research on taxonomy, distribution, pathology and prevention [13], little is known about the phylogeny of T. rubrofasciata in Asia, as DNA sequence information regarding this vector is scarce. While an increasing number of $T$. rubrofasciata have occurred in southern China [6, 9-12], their origins remain unclear. Maximum Likelihood phylogenetic trees inferred by $16 \mathrm{~S}$ rRNA and $28 \mathrm{~S}$ rRNA gene sequences suggest that the Triatoma could be divided into four clades, not three as suggested by a previous study that used a limited sample size [9], and that the T. rubrofasciata from China, Vietnam and Brazil form a new, cohesive clade, indicating a close relationship between T. rubrofasciata from Asia and South America, with no incipient speciation between them.

Though the interrelationships among genera and species of the Triatomini have been shown as unstable in previous studies [43, 44], most researchers consider the Asian species of $T$. rubrofasciata to be derived from the Americas and form a monophyletic clade within the Triatominae $[4,44]$, which is consistent with the findings from this study. The results of the comparative analyses of the 16S rRNA, 28rRNA and COI sequences of $T$. rubrofasciata exhibited high genetic similarity, suggesting a very recent dispersal of this invasive insect in Asia (including China and Vietnam) and a derivation of the insect from South America, primarily from Brazil.

Though this study does allow a better understanding of the phylogenetic relationships among triatomine species and determine the phylogenetic origin approximation of $T$. rubrofasciata in China, extensive 


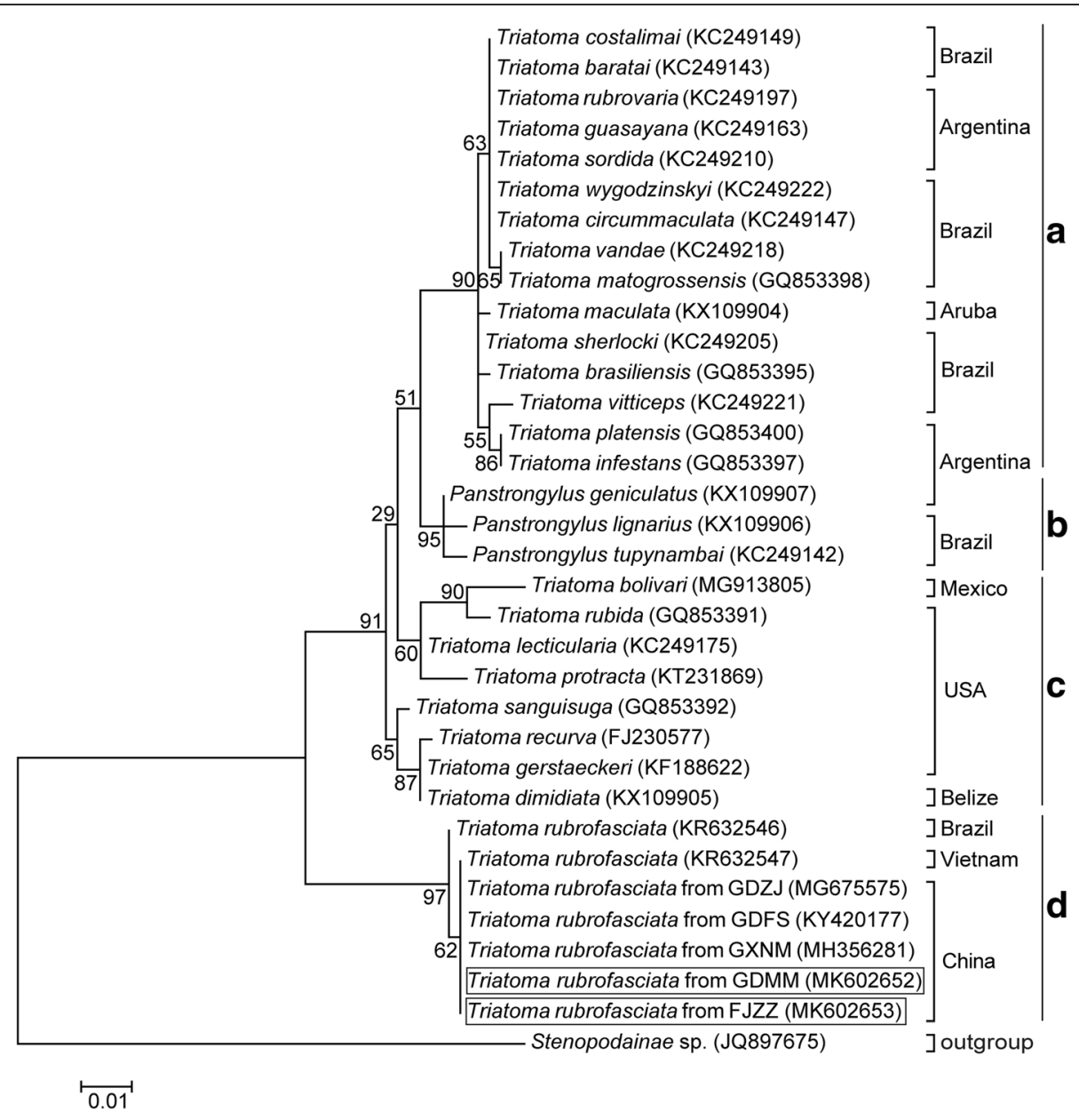

Fig. 5 Phylogenetic tree of $28 \mathrm{~S}$ rRNA gene among triatomine species. The tree was constructed by using the Maximum Likelihood method based on the Kimura 2-Parameter model. The relative values (\%) on branches are based on 1000 bootstrap resamplings. Stenopodainae sp. was included in the trees as the outgroup. T. rubrofasciata identified in this study were shown in the square frame

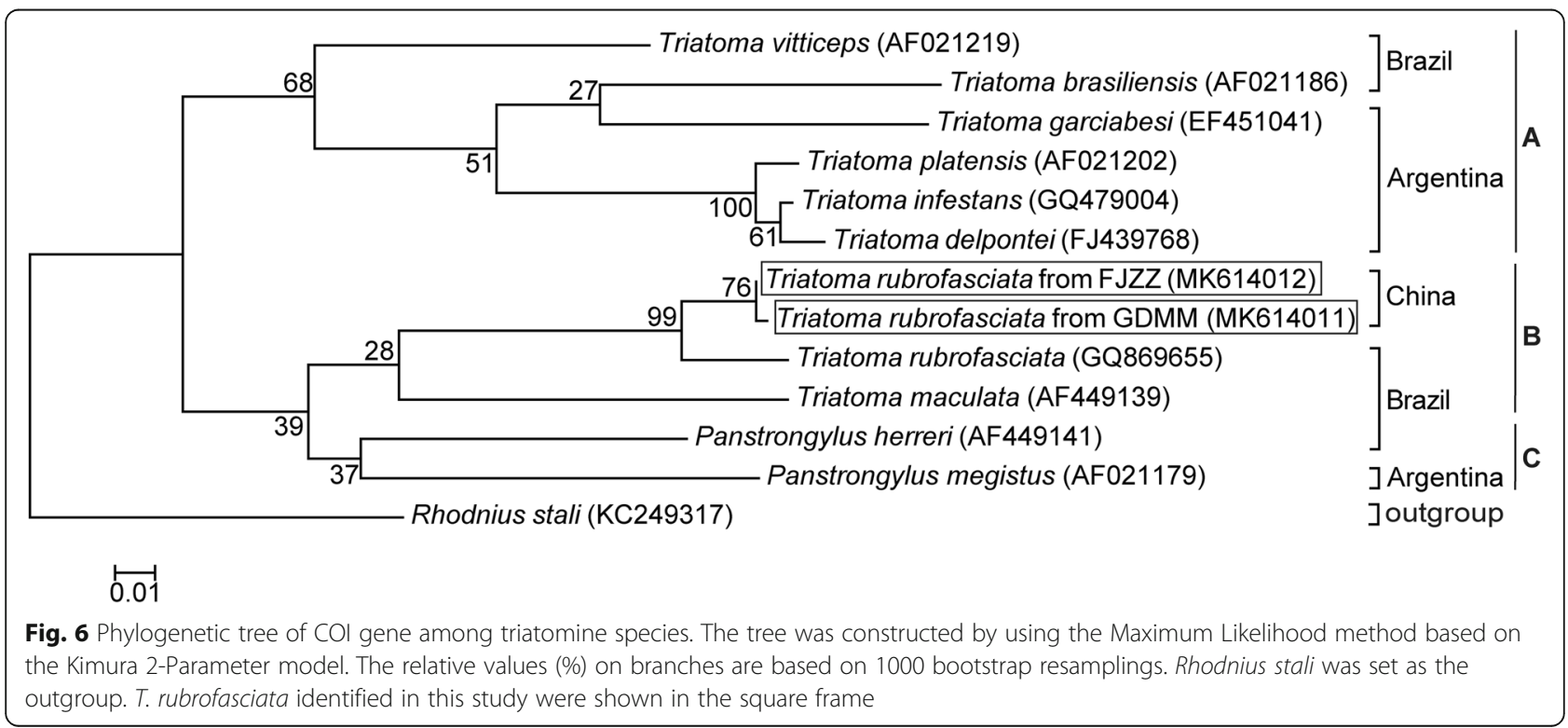


population genetic study is needed to confirm these results due to the small sample size and limited sampling area in this paper. Especially for the COI gene, we can only obtain the sequences of $T$. rubrofasciata from FJZZ and GDMM in China as well as Brazil, but not Vietnam, from NCBI GenBank database. Moreover, the morphological difference between the bugs from China, Vietnam or Brazil is missed in the present study because the authors have not the samples of T. rubrofasciata captured in Vietnam and Brazil.

\section{Conclusions}

This is the first report of the triatomine species found in GDMM and FJZZ in China, which likely originated in Vietnam or Brazil. The current molecular taxonomic study and phylogenetic origin analysis revealed genetic data of $T$. rubrofasciata from different localities in China, which could contribute to the establishment of adequate vector control strategies and adding phylogenetic information regarding this widespread, invasive insect of medical significance.

\section{Additional files}

Additional file 1: Multilingual abstracts in the five official working languages of the United Nations. (PDF 597 kb)

Additional file 2: Table S1. Genetic distance table for $16 \mathrm{~S}$ rRNA sequences used in Fig. 4. Standard error estimates were shown above the diagonal. Analyses were conducted using the Kimura 2-Parameter model. (XLSX $26 \mathrm{~kb}$ )

Additional file 3: Table S2. Genetic distance table for $28 \mathrm{~S}$ rRNA sequences used in Fig. 5. Standard error estimates were shown above the diagonal. Analyses were conducted using the Kimura 2-Parameter model. (XLSX $20 \mathrm{~kb}$ )

Additional file 4: Table S3. Genetic distance table for $\mathrm{COI}$ sequences used in Fig. 6. Standard error estimates were shown above the diagonal. Analyses were conducted using the Kimura 2-Parameter model. (XLSX $11 \mathrm{~kb}$ )

\section{Abbreviations \\ COI: cytochrome oxidase subunit l; FJZZ: Zhangzhou City, Fujian Province, China; GDFS: Foshan City, Guangdong Province, China; GDMM: Maoming City, Guangdong Province, China; GDZJ: Zhanjiang City, Guangdong Province, China; GXNM: Ningming County, Guangxi Province, China; ITS- 2: internal transcribed spacer 2; LAC: Latin American and Caribbean; ML: Maximum Likelihood; NCBI: National Center for Biotechnology Information; PCR: polymerase chain reaction; rRNA: ribosomal RNA; TW: Taiwan}

\section{Acknowledgements}

We are grateful to Prof. Guo-Sheng He, Mr. He-Ming Huang, Mr. Jun Luo and Mrs. Lin-lin Guo for collecting the bugs.

\section{Authors' contributions}

LZY and XHG conceived and designed the experiments and draft the manuscript; HY participated in drafting the manuscript; GMZ collected the samples; HY, GMZ and HP performed the experiments; HY and GMZ collected and analyzed the data; ZHL, MYB, ZMY and CSY participated in study design, technological guidance and coordination. All authors read and approved the final version of the manuscript.

\section{Funding}

This work was supported by grants from the National Key Research and Development Program of China (grant No. 2016 YFC1202003 and 2016YFC1202005), the Project of Basic Platform of National Science and Technology Resources of the Ministry of Sciences and Technology of China (grant No. TDRC-2017-22), the National Natural Science Foundation of China (grant No. 81371836 and 81572023), Science and Technology Planning Project of Guangdong Province (grant No. 2019B030316025), Guangdong Natural Science Foundation (grant No. 2014A030313134), Science and Technology Planning Project of Guangzhou (grant No. 201607010029), the 111 Project (grant No. B12003), the Undergraduates Innovation Training Program of Guangdong Province (grant No. 201410558274 and 201601084) and Teaching Reform Project of Sun Yatsen University (grant no. 2016012).

\section{Availability of data and materials}

Gene data in the present study are being submitted to the National Center for Biotechnology Information (NCBI).

\section{Ethics approval and consent to participate}

Not applicable.

\section{Consent for publication}

Not applicable.

\section{Competing interests}

The authors declare that they have no competing interests.

\section{Author details}

'Zhongshan School of Medicine, Sun Yat-sen University, Guangzhou 510080, China. ${ }^{2}$ Key Laboratory of Tropical Disease Control (Sun Yat-sen University), Ministry of Education, Guangzhou 510080, China. ${ }^{3}$ Provincial Engineering Technology Research Center for Biological Vector Control, Guangzhou 510080, China. ${ }^{4}$ Department of Gastroenterology of the Fifth Affiliated Hospital, Sun Yat-sen University, Zhuhai 519000, China. ${ }^{5}$ Fujian Center for Disease Control and Prevention, Fuzhou 350001, China.

Received: 6 March 2019 Accepted: 19 July 2019

Published online: 13 August 2019

\section{References}

1. World Health Organization: Chagas disease (American trypanosomiasis). https://www.who.int/chagas/epidemiology/en/. Accessed 17 Apr 2019

2. Dye-Braumuller KC, Gorchakov R, Gunter SM, Nielsen DH, Roachell WD, Wheless $\mathrm{A}$, et al. Identification of Triatomines and their habitats in a highly developed urban environment. Vector Borne Zoonotic Dis. 2019;19:265-73.

3. Gourbière S, Dorn P, Tripet F, Dumonteil E. Genetics and evolution of triatomines: from phylogeny to vector control. Heredity (Edinb). 2012;108:190-202.

4. Galvão C, Justi SA. An overview on the ecology of Triatominae (Hemiptera: Reduviidae). Acta Trop. 2015;151:116-25.

5. Anderson C, Belnap C. The kiss of death: a rare case of anaphylaxis to the bite of the "red margined kissing bug". Hawaii J Med Public Health. 2015;74:33-5.

6. Liu Q, Guo YH, Zhang Y, Zhou ZB, Zhang LL, Zhu D, et al. First records of Triatoma rubrofasciata (De Geer, 1773) (Hemiptera, Reduviidae) in Foshan, Guangdong Province. Southern China Infect Dis Poverty. 2017;6:129.

7. Cortéz MG, Gonçalves TC. Resistance to starvation of Triatoma rubrofasciata (De Geer, 1773) under laboratory conditions (Hemiptera: Reduviidae: Triatominae). Mem Inst Oswaldo Cruz. 1998:9:549-54.

8. Bayer AM, Hunter GC, Gilman RH, Cornejo Del Carpio JG, Naquira C, Bern C, et al. Chagas disease, migration and community settlement patterns in Arequipa. Peru PLoS Negl Trop Dis. 2009;3:e567.

9. Huang $Y L$, Huang DN, Wu WH, Yang F, Zhang XM, Wang $M$, et al. Identification and characterization of the causative triatomine bugs of anaphylactic shock in Zhanjiang. China Infect Dis Poverty. 2018;7:127.

10. Chen MX. The prevention and treatment of the dermatitis caused by triatomine bites. Hainan Med J. 1986;14:35 (in Chinese)

11. Wang LY, Peng LF. Four cases of anaphylactic shock caused by triatomine bugs. Clin Focus. 2006;21:1059-61 (in Chinese).

12. Liu Q, Zhou XN. Preventing the transmission of American trypanosomiasis and its spread into non-endemic countries. Infect Dis Poverty. 2015;4:60. 
13. Dujardin JP, Lam TX, Khoa PT, Schofield CJ. The rising importance of Triatoma rubrofasciata. Mem Inst Oswaldo Cruz. 2015;110:319-23.

14. Qian YJ, Li SZ, Wang Q, Zhang L, Liu W, Chen JX, et al. Rapid risk assessment on the import of American trypanosomiasis to China. Chin J Parasitol Parasit Dis. 2013;31:57-9 (in Chinese).

15. Mendonça VJ, Alevi KC, Medeiros LM, Nascimento JD, de Azeredo-Oliveira MT, da Rosa JA. Cytogenetic and morphologic approaches of hybrids from experimental crosses between Triatoma lenti Sherlock \& Serafim, 1967 and T. sherlocki papa et al., 2002 (Hemiptera: Reduviidae). Infect Genet Evol. 2014;26:123-31.

16. Freitas SP, Gonçalves TC, Serrão JE, Costa J, Santos-Mallet JR. Male reproductive system structure and accessory glands ultrastructure of two species of Triatoma (Hemiptera, Reduviidae, Triatominae). Micron. 2010;41:518-25.

17. Rivas N, Sánchez-Cordero V, Camacho AD, Alejandre-Aguilar R. External female genitalia of six species of the genus Meccus (Hemiptera: Reduviidae: Triatominae). J Vector Ecol. 2017:42:271-8.

18. Tellez-Garcia AA, Bello-Bedoy R, Enríquez-Vara JN, Córdoba-Aguilar A, GutiérrezCabrera AE. Genital morphology and copulatory behavior in triatomine bugs (Reduviidae: Triatominae). Arthropod Struct Dev. 2019;49:103-18.

19. Sheir SK, Galal-Khallaf A, Mohamed AH, Mohammed-Geba K. Morphological and molecular clues for recording the first appearance of Artemia franciscana (Kellogg, 1906) in Egypt. Heliyon. 2018;4:e01110.

20. Lee EH, Park SH, Eo JK, Ka KH, Eom AH. Acaulosproa koreana, a new species of arbuscular mycorrhizal fungi (Glomeromycota) associated with roots of woody plants in Korea. Mycobiology. 2018;46:341-8.

21. Lass-Flörl C. How to make a fast diagnosis in invasive aspergillosis. Med Mycol. 2019;57:S155-60.

22. Zhang B, Wang L, Liu J, Xu L, Song L, Wu X, et al. Case report: a rare case of urinary myiasis induced by the fourth instar larvae of Telmatoscopus albipunctatus. PLoS Negl Trop Dis. 2017;11:e0006016.

23. Martínez FH, Villalobos GC, Cevallos AM, Torre Pde L, Laclette JP, AlejandreAguilar R, et al. Taxonomic study of the Phyllosoma complex and other triatomine (Insecta: Hemiptera: Reduviidae) species of epidemiological importance in the transmission of Chagas disease: using ITS-2 and mtCytB sequences. Mol Phylogenet Evol. 2006;41:279-87.

24. Almeida CE, Faucher L, Lavina M, Costa J, Harry M. Molecular individualbased approach on Triatoma brasiliensis: inferences on Triatomine Foci, Trypanosoma cruzi natural infection prevalence, parasite diversity and feeding sources. PLoS Negl Trop Dis. 2016;10:e0004447.

25. Marcilla A, Bargues MD, Ramsey JM, Magallon-Gastelum E, Salazar-Schettino PM, Abad-Franch F, et al. The ITS-2 of the nuclear rDNA as a molecular marker for populations, species, and phylogenetic relationships in Triatominae (Hemiptera: Reduviidae), vectors of Chagas disease. Mol Phylogenet Evol. 2001;18:136-42.

26. Espinoza B, Martínez-Ibarra JA, Villalobos G, De La Torre P, Laclette JP, Martínez-nández F. Genetic variation of north American Triatomines (Insecta: Hemiptera: Reduviidae): initial divergence between species and populations of Chagas disease vector. Am J Trop Med Hyg. 2013;88:275-84.

27. Dorn PL, Justi SA, Dale C, Stevens L, Galvão C, Lima-Cordón R, et al. Description of Triatoma mopan sp. n. from a cave in Belize (Hemiptera, Reduviidae, Triatominae). Zookeys. 2018:775:69-95.

28. de Souza RC, Campolina-Silva GH, Bezerra CM, Diotaiuti L, Gorla DE. Does Triatoma brasiliensis occupy the same environmental niche space as Triatoma melanica? Parasit Vectors. 2015;8:361.

29. Lent H, Wygodzinsky P. Revision of the Triatominae (Hemiptera, Reduviidae), and their significance as vectors of Chagas disease. Bull Am Mus Nat Hist. 1979:163:123-520

30. Altschul SF, Gish W, Miller W, Myers EW, Lipman DJ. Basic local alignment search tool. J Mol Biol. 1990;215:403-10.

31. Larkin MA, Blackshields G, Brown NP, Chenna R, McGettigan PA, Higgins DG. ClustalW and ClustalX version 2.0. Bioinformatics. 2007;21:2947-8.

32. Kumar S, Stecher G, Tamura K. MEGA7: molecular evolutionary genetics analysis version 7.0 for bigger datasets. Mol Biol Evol. 2016;33:1870-4.

33. Kimura M. A simple method for estimating evolutionary rates of base substitutions through comparative studies of nucleotide sequences. J Mol Evol. 1980;16:111-20

34. Braga MV, Pinto ZT, Lima MM. Life cycle and reproductive patterns of Triatoma rubrofasciata (De Geer, 1773) (Hemiptera: Reduviidae), under laboratory conditions. Mem Inst Oswaldo Cruz. 1998;93:539-42.

35. Kalshoven LGE. Observations on the blood-sucking reduviid Triatoma rubrofasciata (De Geer) in Java. Entomologische Berichten. 1970;30:41-7.
36. Vieira CB, Praça YR, Bentes KLDS, Santiago PB, Silva SMM, Silva GDS, et al. Triatomines: Trypanosomatids, Bacteria, and viruses potential vectors? Front Cell Infect Microbiol. 2018;8:405.

37. Gorla DE, Dujardin JP, Schofield CJ. Biosystematics of old world triatominae. Acta Trop. 1997;63:127-40.

38. Klotz SA, Dorn PL, Mosbacher M, Schmidt JO. Kissing bugs in the United States: risk for vector-borne disease in humans. Environ Health Insights. 2014;8:49-59.

39. Martínez F, Alejandre-Aguilar R, Moncada YH, Espinoza B. Molecular taxonomic study of Chagas disease vectors from the phyllosoma, lecticularia, and rubrofasciata complexes. Am J Trop Med Hyg. 2005;73:321-5.

40. Patterson JS, Schofield CJ, Dujardin JP, Miles MA. Population morphometric analysis of the tropicopolitan bug Triatoma rubrofasciata and relationships with old world species of Triatoma: evidence of New World ancestry. Med Vet Entomol. 2001;15:443-51.

41. Ryckman RE, Archbold EF. The Triatominae and Triatominae-borne trypanosomes of Asia, Africa, Australia and the east indies. Bull Soc Vector Ecologists. 1981;6:143-66.

42. Hypsa V, Tietz DF, Zrzavý J, Rego RO, Galvao C, Jurberg J. Phylogeny and biogeography of Triatominae (Hemiptera: Reduviidae): molecular evidence of a New World origin of the Asiatic clade. Mol Phylogenet Evol. 2002;23:447-57.

43. Schofield CJ, Galvão C. Classification, evolution, and species groups within the Triatominae. Acta Trop. 2009:110:88-100.

44. Dujardin JP, Pham Thi K, Truong Xuan L, Panzera F, Pita S, Schofield CJ. Epidemiological status of kissing-bugs in South East Asia: a preliminary assessment. Acta Trop. 2015;151:142-9.

45. Young MR, Moraza ML, Ueckermann E, Heylen D, Baardsen LF, Lima-Barbero $J F$, et al. Linking morphological and molecular taxonomy for the identification of poultry house, soil, and nest dwelling mites in the Western Palearctic. Sci Rep. 2019;9:5784.

46. Chaudhry U, van Paridon B, Lejeune M, Shabbir MZ, Rashid MI, Ashraf K, et al. Morphological and molecular identification of Explanatum explanatum in domestic water buffalo in Pakistan. Vet Parasitol Reg Stud Reports. 2017;8:54-9.

47. Manoylov KM. Taxonomic identification of algae (morphological and molecular): species concepts, methodologies, and their implications for ecological bioassessment. J Phycol. 2014;50:409-4024.

48. Abdullah HHAM, El-Molla A, Salib FA, Allam NAT, Ghazy AA, Abdel-Shafy S. Morphological and molecular identification of the brown dog tick Rhipicephalus sanguineus and the camel tick Hyalomma dromedarii (Acari: Ixodidae) vectors of rickettsioses in Egypt. Vet World. 2016:9:1087-101.

49. Hebert PD, Gregory TR. The promise of DNA barcoding for taxonomy. Syst Biol. 2005;54:852-9.

50. Larsson J. Molecular versus morphological approach to microsporidian classification. Folia Parasitol. 2005;52:143-4.

51. Stepanović S, Kosovac A, Krstić O, Jović J, Toševski I. Morphology versus DNA barcoding: two sides of the same coin. A case study of Ceutorhynchus erysimi and C. contractus identification. Insect Sci. 2016:23:638-48.

52. Mardulyn P, Whitfield JB. Phylogenetic signal in the COI, 165 , and 285 genes for inferring relationships among genera of Microgastrinae (Hymenoptera; Braconidae): evidence of a high diversification rate in this group of parasitoids. Mol Phylogenet Evol. 1999;12:282-94.

53. Klussmann-Kolb A, Dinapoli A, Kuhn K, Streit B, Albrecht C. From sea to land and beyond - new insights into the evolution of euthyneuran Gastropoda (Mollusca). BMC Evol Biol. 2008;8:57.

Ready to submit your research? Choose BMC and benefit from:

- fast, convenient online submission

- thorough peer review by experienced researchers in your field

- rapid publication on acceptance

- support for research data, including large and complex data types

- gold Open Access which fosters wider collaboration and increased citations

- maximum visibility for your research: over $100 \mathrm{M}$ website views per year

At BMC, research is always in progress.

Learn more biomedcentral.com/submission 